\title{
Global Lyapunov functions and a hierarchical control scheme for networks of robotic agents
}

\author{
John Maidens \\ Department of Electrical Engineering \\ \& Computer Sciences \\ University of California, Berkeley \\ Berkeley, CA, 94720, USA \\ Email: maidens@eecs.berkeley.edu
}

\author{
Michael Y. Li \\ Department of Mathematical \& Statistical Sciences \\ University of Alberta \\ Edmonton, AB, T6G 2G1, Canada \\ Email: mli@math.ualberta.ca
}

\begin{abstract}
We present a graph-theoretic method of systematically constructing global Lyapunov functions for large-scale, heterogeneous multi-agent networks with nonlinear dynamics. We illustrate the power of this method by demonstrating the stability of a control protocol for the self-organization of swarms of mobile agents into hierarchical formations.
\end{abstract}

\section{INTRODUCTION}

Recent interest in the distributed, cooperative control of multi-agent systems has generated a wealth of research into stable formation control for systems of autonomous agents [1]-[3]. The formation control problem involves the development of algorithms to control the relative positions and orientations of a group of robots, allowing the group to perform tasks and navigate its environment as a whole [4]. This aggregate behaviour enables the completion of tasks like distributed sensing, monitoring and surveillance to be performed without the guidance of a centralized controller.

Analysis of a formation control algorithm often involves a proof that the algorithm is stable for any initial configuration of the agents [5]-[7]. In this paper, we prove the stability of hierarchically coupled multi-agent systems by demonstrating the existence of a global Lyapunov function.

We provide a general method of constructing Lyapunov functions for multi-agent systems from simple Lyapunov functions $V_{i}$ corresponding to the dynamics of each agent in the system. The results presented here are based on the graph-theoretic approach of Li and Shuai [8], which provides a method of choosing coefficients $c_{i}$ to construct global Lyapunov functions of the form

$$
V(x)=\sum_{i=1}^{n} c_{i} V_{i}\left(x_{i}\right) .
$$

This construction relies on Kirchhoff's Matrix Tree Theorem to choose the coefficients $c_{i}$.

First, we present a combinatorial result, (Theorem 1) that allows us to rearrange terms in the derivative of the Lyapunov function in terms of cycles in the graph. This then leads to a simple criterion for verifying that $\dot{V}$ is non-positive (Theorem 2). Rather than considering the network as a whole, with its multiple coupled feedback paths, Theorem 2 allows us to show that $\dot{V} \leq 0$ by considering only individual feedback loops.
Next, we present a hierarchical formation control protocol which clusters agents into $k$ swarms, and co-ordinates the velocities of these swarms via simple leader-to-leader consensus. We demonstrate the applicability of our results by using them to construct a Lyapunov function that demonstrates the global stability of the closed-loop system.

\section{PRELIMINARIES}

\section{A. Graph-theoretic preliminaries}

A weighted directed graph, or weighted digraph, is a graph $\mathcal{G}=(V(\mathcal{G}), E(\mathcal{G}))$ together with a weight function $\omega: E(\mathcal{G}) \rightarrow \mathbb{R}_{+}$that assigns to each edge of $\mathcal{G}$ a nonnegative weight. We can extend the domain of the weight function $\omega$ to the set of subgraphs of $\mathcal{G}$ by defining the weight of a subgraph as the product of the weights of all edges in the subgraph.

A weighted digraph can be described by its weighted adjacency matrix $A_{\mathcal{G}}$, with entries $a_{j}^{i}$ representing the weight $\omega(\overrightarrow{i j})$ of the edge $\overrightarrow{i j}$ directed from vertex $i$ to vertex $j$. The set of neighbours $\mathcal{N}_{i}=\{j \in V(\mathcal{G}): \overrightarrow{j i} \in E(\mathcal{G})\}$ of vertex $i$ consists of all vertices with incoming connections to $i$. A sequence of edges of $\mathcal{G} i_{1} \rightarrow i_{2} \rightarrow \cdots \rightarrow i_{\ell} \rightarrow i_{1}$ that begins and ends at the same vertex is called a directed cycle, or feedback cycle, of length $\ell$ provided that the vertices $i_{1}, \ldots, i_{\ell}$ are all distinct.

Given an $n$-vertex graph $\mathcal{G}$, a subgraph of $\mathcal{G}$ is called a directed spanning tree rooted at vertex $i$ if it contains $n-1$ edges and a directed path from the root vertex $i$ to each other vertex of $\mathcal{G}$. A spanning unicyclic subgraph of $\mathcal{G}$ is a spanning directed subgraph consisting of a collection of disjoint rooted directed trees whose roots are connected by a directed cycle. For a detailed discussion of these concepts, we refer the reader to [9].

The Laplacian matrix $L_{\mathcal{G}}$ is defined as $L_{\mathcal{G}}=D_{\mathcal{G}}-A_{\mathcal{G}}$ where $D_{\mathcal{G}}$ is the in-degree matrix

$$
D_{\mathcal{G}}=\operatorname{diag}\left(\sum_{j \in V(\mathcal{G})} a_{1}^{j}, \ldots, \sum_{j \in V(\mathcal{G})} a_{M}^{j}\right) .
$$

Since the columns of $L_{\mathcal{G}}$ all sum to zero, the cofactors corresponding to the entries of each column of $L_{\mathcal{G}}$ are all identical. Thus for each $i \in\{1, \ldots, M\}$ we can define the 
Laplacian cofactor $c_{i}$ as the cofactor corresponding to any entry of the $i$-th column of $L_{\mathcal{G}}$. Kirchhoff's Matrix Tree Theorem allows us to express the cofactor $c_{i}$ as the sum of the weights of directed spanning trees rooted at vertex $i$.

Proposition 1 (Kirchhoff's Matrix-Tree Theorem):

Let $\mathbb{T}_{i}$ denote the set of directed spanning subtrees of $\mathcal{G}$ rooted at vertex $i$. For each vertex $i \in V(\mathcal{G})$ we have

Proof: See [9], [10].

$$
c_{i}=\sum_{\mathcal{T} \in \mathbb{T}_{i}} \omega(\mathcal{T})
$$

\section{B. Multi-agent systems}

A system of $n$ agents is modeled by assigning to each agent $i$ a nonlinear equation

$$
\dot{x}_{i}=f_{i}\left(x_{i}\right) \quad x_{i} \in \mathbb{R}^{m_{i}}
$$

describing the agent's internal dynamics. Agents are assumed to interact via a coupling function $g_{i}^{j}(x)$ that represents the influence of agent $j$ on agent $i$. Each agent then corresponds to a vertex of an $n$-vertex graph $\mathcal{G}$ that describes the interaction between agents with an edge directed from vertex $j$ to vertex $i$ whenever $g_{i}^{j}$ is not identically zero. The coupled $M$-dimensional system can then be written as

$$
\dot{x}_{i}=f_{i}\left(x_{i}\right)+\sum_{j \in \mathcal{N}_{i}} g_{i}^{j}(x), \quad x \in D
$$

where $x=\left(x_{1}, \ldots, x_{n}\right), D$ is an open set in $\prod_{i=1}^{n} \mathbb{R}^{m_{i}}$ and $M=\sum_{i=1}^{n} m_{i}$ We assume that the functions $f_{i}$ and $g_{i}^{j}$ are sufficiently well-behaved to guarantee the existence and uniqueness of solutions to (2).

Our goal is to systematically construct a global Lyapunov function $V: D \rightarrow \mathbb{R}$ for the aggregate system (2) as a linear combination of Lyapunov candidate functions $V_{i}$ corresponding to each agent $i$, ensuring that the time derivative $\dot{V}(x)=\nabla V \cdot f(x)$ of the aggregate Lyapunov function along trajectories of the system is non-positive everywhere in $D$.

\section{GLOBAL LyAPUNOV FUNCTIONS FOR NETWORKED SYSTEMS}

\section{A. Tree cycle identity}

Consider a weighted digraph $\mathcal{G}$ with non-negative weights $a_{i}^{j}$. Assign to each edge $\overrightarrow{j i}$ an arbitrary edge function $F_{j}^{i}$ : $\mathbb{R}^{M} \rightarrow \mathbb{R}$. The vertex function for vertex $i$ is then given by

$$
F_{i}(x)=\sum_{j \in \mathcal{N}_{i}} a_{i}^{j} F_{i}^{j}(x) .
$$

We wish to assign weights $w_{i}$ to each vertex $i$ such that the weighted sum

$$
H(x)=\sum_{i \in V(\mathcal{G})} w_{i} F_{i}(x)
$$

can be expressed in terms of feedback cycles in $\mathcal{G}$. This will allow us to determine properties of the overall sum $H$ based on properties of smaller sums around each feedback cycle, which are much easier to verify in practical applications. To develop a method of choosing these vertex weights, we

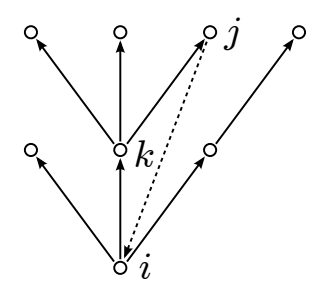

(a) Spanning unicyclic graph formed from a directed spanning tree rooted at vertex $i$ by adding an edge directed from vertex $j$ to $i$

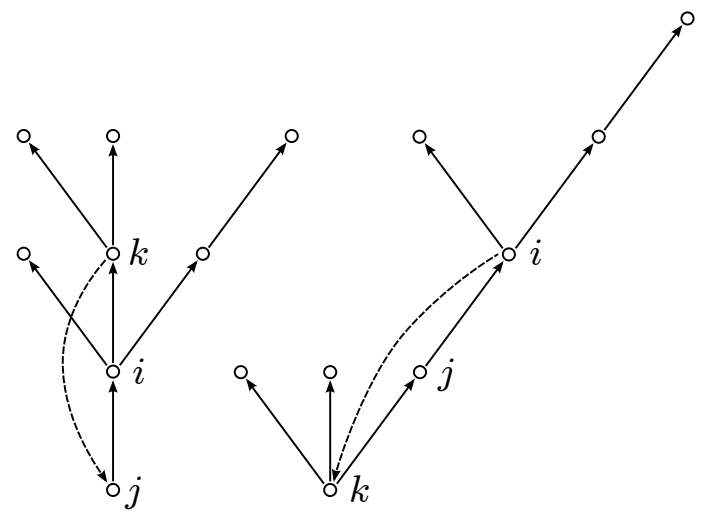

(b) The same spanning unicyclic graph shown can be formed in two other ways, from directed spanning trees rooted at vertices $j$ and $k$.

Fig. 1: Construction of spanning unicyclic graphs as the union of a directed spanning tree and a single edge

consider unicyclic subgraphs of $\mathcal{G}$. We denote the set of all spanning unicyclic subgraphs of $\mathcal{G}$ by $\mathbb{P}$.

An element $\mathcal{P}$ of $\mathbb{P}$ with cycle length $\ell$ can be expressed in precisely $\ell$ distinct ways as the union of a directed spanning tree and a single directed edge (see Figure 1). Indeed, removing each edge $\overrightarrow{j i}$ from the cycle $\mathcal{C}_{\mathcal{P}}$ of $\mathcal{P}$ yields a directed spanning tree rooted at vertex $i$.

In the following theorem, we show that by choosing vertex weights $w_{i}$ equal to the cofactors $c_{i}$ of the weighted Laplacian matrix $L_{\mathcal{G}}$ we can re-organize the weighted sum $H$ in terms of feedback cycles in $\mathcal{G}$.

Theorem 1 (Tree Cycle Identity):

Let $c_{i}$ be given as in (1). Then

$$
H(x)=\sum_{i \in V(\mathcal{G})} c_{i} F_{i}(x)=\sum_{\mathcal{P} \in \mathbb{P}} \omega(\mathcal{P}) \sum_{\vec{j} i \in E\left(\mathcal{C}_{\mathcal{P}}\right)} F_{i}^{j}(x)
$$

Proof: By Proposition 1

$$
c_{i}=\sum_{\mathcal{T} \in \mathbb{T}_{i}} \omega(\mathcal{T}) .
$$

Then

$$
\begin{aligned}
\sum_{i \in V(\mathcal{G})} c_{i} F_{i}(x) & =\sum_{i \in V(\mathcal{G})}\left(\sum_{\mathcal{T} \in \mathbb{T}_{i}} \omega(\mathcal{T})\right)\left(\sum_{j \in \mathcal{N}_{i}} a_{i}^{j} F_{i}^{j}(x)\right) \\
& =\sum_{i \in V(\mathcal{G})} \sum_{\mathcal{T} \in \mathbb{T}_{i}} \sum_{j \in \mathcal{N}_{i}} a_{i}^{j} \omega(\mathcal{T}) F_{i}^{j}(x) .
\end{aligned}
$$

Now, for each triple $(i, j, \mathcal{T})$ with $i \in V(\mathcal{G}), j \in \mathcal{N}_{i}$ and $\mathcal{T} \in \mathbb{T}_{i}$ there is a unique spanning unicyclic graph $\mathcal{P} \in \mathbb{P}$ formed from $\mathcal{T}$ and the edge $\overrightarrow{j i}$. Conversely, for each pair 
$(Q, \overrightarrow{j i})$ with $Q \in \mathbb{P}$ and $\overrightarrow{j i} \in E\left(\mathcal{C}_{\mathcal{P}}\right)$ there is a unique $\mathcal{T} \in \mathbb{T}_{i}$ resulting from the removal of edge $\overrightarrow{j i}$. Thus there is a direct correspondence between triples of the form $(i, j, \mathcal{T})$ and pairs of the form $(Q, \overrightarrow{j i})$. Then noting that $a_{i}^{j} \omega(\mathcal{T})=$ $\omega(\mathcal{P})$, we see that

$$
\begin{aligned}
H(x) & =\sum_{i \in V(\mathcal{G})} \sum_{\mathcal{T} \in \mathbb{T}_{i}} \sum_{j \in \mathcal{N}_{i}} a_{i}^{j} \omega(\mathcal{T}) F_{i}^{j}(x) \\
& =\sum_{\mathcal{P} \in \mathbb{P}} \omega(\mathcal{P}) \sum_{\overrightarrow{j i} \in E\left(\mathcal{C}_{\mathcal{P}}\right)} F_{i}^{j}(x) .
\end{aligned}
$$

\section{B. Constructing Lyapunov functions via feedback cycles}

Constructing Lyapunov functions $V$ for large-scale networked systems is difficult due to the unwieldy number of terms in the Lyapunov derivative of $V$ with respect to system (2). Even when each of the $n$ agents is assigned only a single state variable, the number of terms in the expression

$$
\dot{V}(x)=\sum_{i \in V(\mathcal{G})} \frac{\partial V}{\partial x_{i}}\left(f_{i}\left(x_{i}\right)+\sum_{j \in V(\mathcal{G})} g_{i}^{j}(x)\right)
$$

varies as the square of $n$.

Theorem 1 provides us with a method of grouping terms in the Lyapunov function's derivative in terms of cycles in $\mathcal{G}$. This gives us an easily-verifiable condition to guarantee that $\dot{V}(x) \leq 0$ for all $x$.

Definition 1: We say that the set of edge functions $\left\{F_{i}^{j}\right.$ : $i, j \in V(\mathcal{G})\}$ satisfies the cycle condition if, for any directed cycle $\mathcal{C}$ in $\mathcal{G}$, we have

$$
\sum_{\vec{j} i \in E(\mathcal{C})} F_{i}^{j}(x) \leq 0 \quad x \in D .
$$

Theorem 2: Consider a Lyapunov candidate function of the form

$$
V(x)=\sum_{i \in V(\mathcal{G})} c_{i} V_{i}(x)
$$

where each $V_{i}: \mathbb{R}^{M} \rightarrow \mathbb{R}$ is a function corresponding to agent $i$ and $c_{i}$ is given as in Proposition 1. Suppose that there exists a set of edge functions $\left\{F_{i}^{j}: i \in V(\mathcal{G}), j \in \mathcal{N}_{i}\right\}$ such that

1) we have

$$
\dot{V}_{i}(x) \leq F_{i}(x)=\sum_{j \in \mathcal{N}_{i}} a_{i}^{j} F_{i}^{j}(x) \quad x \in D
$$

and

2) the set of edge functions satisfies the cycle condition given in Definition 1.

Then $V$ satisfies $\dot{V}(x) \leq 0$ for all $x \in D$. Namely, $V$ is a Lyapunov function for the system (2).

Proof: By Theorem 1 we have

$$
\begin{aligned}
\dot{V}(x) & \leq \sum_{i \in V(\mathcal{G})} c_{i} \sum_{j \in \mathcal{N}_{i}} a_{i}^{j} F_{i}^{j}(x) \\
& =\sum_{\mathcal{P} \in \mathbb{P}} \omega(\mathcal{P}) \sum_{j i \in E\left(\mathcal{C}_{\mathcal{P}}\right)} F_{i}^{j}(x) \leq 0 .
\end{aligned}
$$

\section{A GLOBALly STABLE HIERARCHICAL FORMATION CONTROL FOR MULTI-AGENT SWARMS}

\section{A. Summary}

Hierarchical approaches to designing communication topologies have previously been considered for linear systems, and issues related to stability have been discussed, see for example [11], [12]. In this section, we provide a nonlinear example of a hierarchical control scheme.

In [13], [14], Tanner, Jadbabaie and Pappas provide a method of stabilizing swarms of agents using artificial potential functions [15], [16]. Their analysis uses a Lyapunov function to demonstrate that their protocol stabilizes the formation at a local minimum of the artificial potentials and leads to the alignment of all agents. Communication networks among agents are either complete (i.e. every agent communicates with every other agent) or dynamic (i.e. communication links appear and disappear based on proximity of agents).

We propose a hierarchical control scheme that requires a reduced number of edges in the communication network compared with the complete networks considered in [13], [14]. The key steps of our hierarchical potential clustering (HPC) protocol are:

1) run a clustering algorithm based on the initial positions of the agents

2) assign leaders to each cluster

3) implement the artificial potential scheme (with complete interaction graph) within each cluster and

4) implement a velocity consensus scheme between the cluster leaders.

\section{B. Implementation of the HPC protocol}

We model each agent by specifying its position $r=$ $(x, y) \in \mathbb{R}^{2}$ and velocity $v=\dot{r}$. The dynamics of each agent are governed by the 4-dimensional system

$$
\begin{aligned}
& \dot{r}=v, \\
& \dot{v}=u,
\end{aligned}
$$

where the thrust $u \in \mathbb{R}^{2}$ is the control input.

Before designing the control inputs, agents are clustered based on their position using an algorithm seeking to minimize the sum-squared distance within each cluster. See [17], [18] for a review of statistical clustering algorithms. If we label the resulting clusters with integers $1, \ldots, N$ then the clustering induces a vector labelling of the agents $(i, j)$ where $i$ is the cluster index and $j \in\left\{1, \ldots, n_{i}\right\}$ is used to distinguish agents within cluster $i$. We use the index $(i, 1)$ to designate the leader of cluster $i$. Clustering algorithms such as the one considered in [19] provide a natural choice of leader called an exemplar. For algorithms that do not provide a leader, one can be assigned arbitrarily. The choice of a leader is not important for our purposes since we will show that stability is independent of the choice of leader. With this labelling of vertices, we obtain a system that describes the collection of agents 


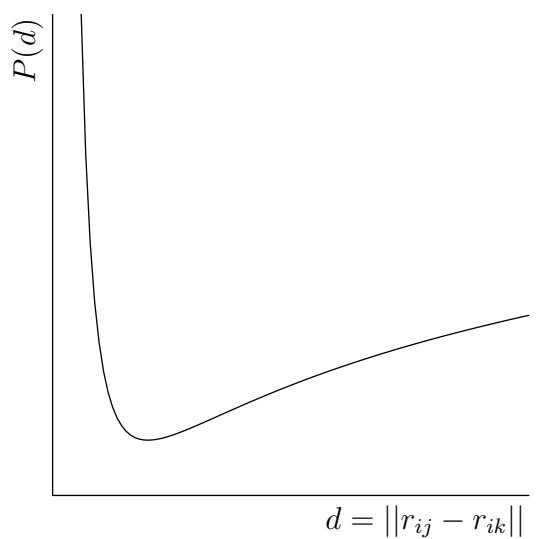

Fig. 2: Artificial potential function $P(d)=\frac{1}{d^{2}}+\log d^{2}$.

$$
\begin{aligned}
& \dot{r}_{i j}=v_{i j} \\
& \dot{v}_{i j}=u_{i j} .
\end{aligned}
$$

Now that we have clustered the agents, we need to design control inputs $u$ to bring each cluster of agents into formation. Within each cluster $i$, we implement the artificial potential control scheme. For each pair of agents $(i, j)$ and $(i, k)$ in cluster $i$ we define an artificial potential function $P_{i j}^{i k}: \mathbb{R}_{+} \rightarrow \mathbb{R}$. The potentials $P_{i j}^{i k}$ can be chosen to be any positive, continuous functions of the distance $d=\left\|r_{i j}-r_{i k}\right\|$ between agents $(i, j)$ and $(i, k)$ that are:

- $\operatorname{symmetric}\left(P_{i j}^{i k}=P_{i k}^{i j}\right)$

- unbounded near zero and infinity $\left(\lim _{d \rightarrow 0, \infty} P_{i j}^{i k}(d)=\infty\right)$

- and that have a unique minimum at the desired distance between agents.

An example of an appropriate potential function is shown in Figure 2. We can then define the total potential for vertex $(i, j)$ as

$$
P_{i j}=\sum_{k=1}^{n_{i}} P_{i j}^{i k}
$$

Now, for each pair of vertices $(i, j)$ and $(i, k)$ from the same cluster, define the function $u_{i j}^{i k}: \mathbb{R}^{2} \times \mathbb{R}^{2} \rightarrow \mathbb{R}$ as $u_{i j}^{i k}\left(r_{i j}, r_{i k}\right)=\left\|r_{i j}-r_{i k}\right\|$. The position derivative $\nabla_{r_{i j}}$ of the total potential function $P_{i j}$ is then defined as the 2dimensional vector

$$
\nabla_{r_{i j}} P_{i j}=\sum_{k=1}^{n_{i}}\left(\frac{\partial\left(P_{i j}^{i k} \circ u_{i j}^{i k}\right)}{\partial x_{i j}}, \frac{\partial\left(P_{i j}^{i k} \circ u_{i j}^{i k}\right)}{\partial y_{i j}}\right) .
$$

This notation, used in [13], [14], emphasizes the fact that the control protocol given in the following paragraph is essentially a modified gradient descent, in which each agent seeks to minimize its total potential using the local information available to it. The artificial potential control protocol combines this gradient descent with a consensus protocol in the heading $\theta_{i j}$ as follows:

$$
u_{i j}=-\nabla_{r_{i j}} P_{i j}-\sum_{k} \frac{\left(\theta_{i j}-\theta_{i k}\right)\left\|v_{i j}\right\|}{\left\|r_{i j}-r_{i k}\right\|} \hat{n}(i j) \quad j \neq 1
$$

where $\hat{n}(i j)$ is the unit vector orthogonal to $v_{i j}$, and $\theta_{i j}=$ $\operatorname{atan} 2\left(\dot{y}_{i j}, \dot{x}_{i j}\right)$ is the heading of agent $(i, j)$. Among the leaders of each cluster we apply an additional thrust to coordinate their velocities

$$
\begin{aligned}
u_{i 1}= & -\nabla_{r_{i 1}} P_{i 1}-\sum_{k} \frac{\left(\theta_{i 1}-\theta_{i k}\right)\left\|v_{i 1}\right\|}{\left\|r_{i 1}-r_{i k}\right\|} \hat{n}(i 1) \\
& +\sum_{h \in N_{i}} b_{i h}\left(v_{h 1}-v_{i 1}\right)
\end{aligned}
$$

where $b_{i h} \geq 0$ are the entries of an irreducible matrix $B$. This ensures that the leader communication graph $\mathcal{G}_{B}$ that encodes the interactions among the cluster leaders is strongly connected [20].

\section{Proof of stability}

We wish to demonstrate that the system defined by Equation (3) will eventually reach a desirable equilibrium.

Definition 2: A control protocol $u$ is said to solve the formation stabilization problem if solutions of (3) converge asymptotically to a state in which

1) the relative positions of each agent $(i, j)$ within a cluster are such that a local minimum of the total vertex potential $P_{i j}$ is achieved, and

2) for any two agents $(i, j)$ and $(h, k)$, their headings satisfy $\theta_{i j}=\theta_{h k}$.

The following lemma is needed in the proof of our main theorem. Its proof is the same as that for Equation (7) in [14] and is therefore omitted.

Lemma 1:

$$
\dot{\theta}_{i j}=-\sum_{k=1}^{n_{i}} \frac{\theta_{i j}-\theta_{i k}}{\left\|r_{i j}-r_{i k}\right\|^{2}} .
$$

Theorem 3: Given any clustering scheme, the HPC protocol solves the formation stabilization problem provided that the leader communication graph $\mathcal{G}_{B}$ is strongly connected.

Proof: For each agent indexed $(i, j)$, define a vertex Lyapunov function

$$
V_{i j}=\frac{1}{2}\left(\sum_{k=1}^{N_{i}} P_{i j}^{i k}\left(\left\|r_{i j}-r_{i k}\right\|\right)+v_{i j} \cdot v_{i j}+\theta_{i j}^{2}\right)
$$

and let $c=\left(c_{1}, \ldots, c_{N}\right)$ denote the vector of cofactors of the columns of $L_{\mathcal{G}_{B}}$, as given by Kirchhoff's Matrix Tree Theorem. Define

$$
V=\sum_{i=1}^{N} c_{i} \sum_{j=i}^{n_{i}} V_{i j}
$$

We will show that $V$ is a Lyapunov function for the multiagent system (3). Since $\mathcal{G}_{B}$ is strongly connected, there is a tree rooted at each vertex of $\mathcal{G}_{B}$. Thus all entries of $c$ are strictly positive and hence $V$ is positive definite. Taking the 
derivative of $V$ along trajectories of (3) we get

$$
\begin{aligned}
\dot{V}= & \sum_{i=1}^{N} c_{i} \sum_{j=i}^{n_{i}} \dot{V}_{i j} \\
= & \sum_{i=1}^{N} c_{i} \sum_{j=i}^{n_{i}} \frac{1}{2}\left[\sum_{k=1}^{n_{i}}\left(\nabla_{r_{i j}} P_{i j}^{i k} \cdot \dot{r}_{i j}+\nabla_{r_{i k}} P_{i j}^{i k} \cdot \dot{r}_{i k}\right)\right. \\
& \left.+2 v_{i j} \cdot \dot{v}_{i j}+2 \theta_{i j} \dot{\theta}_{i j}\right]
\end{aligned}
$$

Since we assume that the artificial potentials are symmetric, (i.e. $P_{i j}^{i k}=P_{i k}^{i j}$ ) we can re-write this expression for $\dot{V}$.

$$
\begin{aligned}
\dot{V}= & \sum_{i=1}^{N} c_{i} \sum_{j=i}^{n_{i}}\left[\sum_{k=1}^{n_{i}} \nabla_{r_{i j}} P_{i j}^{i k} \cdot \dot{r}_{i j}+v_{i j} \cdot \dot{v}_{i j}+\theta_{i j} \dot{\theta}_{i j}\right] \\
= & \sum_{i=1}^{N} c_{i} \sum_{j=i}^{n_{i}}\left[\nabla_{r_{i j}} P_{i j} \cdot v_{i j}\right. \\
& +v_{i j} \cdot\left(-\nabla_{r_{i j}} P_{i j}-\sum_{k} \frac{\left(\theta_{i j}-\theta_{i k}\right)\left\|v_{i j}\right\|}{\left\|r_{i j}-r_{i k}\right\|} \hat{n}(i j)\right) \\
& \left.+v_{i 1} \cdot \sum_{h \in \mathcal{N}_{i}} b_{i h}\left(v_{h 1}-v_{i 1}\right)+\theta_{i j} \dot{\theta}_{i j}\right]
\end{aligned}
$$

By definition, the vector $\hat{n}(i j)$ is orthogonal to $v_{i j}$ so we can further simplify our expression for $\dot{V}$.

$$
\dot{V}=\sum_{i=1}^{N} c_{i} \sum_{j=i}^{n_{i}} \theta_{i j} \dot{\theta}_{i j}+\sum_{i=1}^{N} c_{i} \sum_{h \in \mathcal{N}_{i}} b_{i h}\left(v_{h 1}-v_{i 1}\right) \cdot v_{i 1}
$$

For each cluster $i$, define the cluster distance graph $\mathcal{G}_{i}$ as the complete graph on $n_{i}$ vertices (without self-loops) where the edge $\overrightarrow{j k}$ is assigned a weight $\frac{1}{\left\|r_{i j}-r_{i k}\right\|^{2}}$ corresponding to the squared distance between agents $(i, j)$ and $(i, k)$. We can also define the vector $\theta_{i}=\left(\theta_{i 1}, \ldots, \theta_{i n_{i}}\right)^{T} \in \mathbb{R}^{n_{i}}$ to be the concatenation of the headings of all agents in group $i$. Using Lemma 1 we can rewrite the expression for $V$ in terms of the weighted Laplacian matrix $L_{i}$ of the graph $\mathcal{G}_{i}$ as follows

$$
\dot{V}=-\sum_{i=1}^{N} c_{i} \theta_{i}^{T} L_{i} \theta_{i}+\sum_{i=1}^{N} c_{i} \sum_{h \in \mathcal{N}_{i}} b_{i h}\left(v_{h 1}-v_{i 1}\right) \cdot v_{i 1}
$$

For each group $i$, the Laplacian $L_{i}$ is symmetric and positive semidefinite. Since $\mathcal{G}_{i}$ is strongly connected, the nullspace of $L_{i}$ is spanned by the consensus vector $\mathbf{1}=(1, \ldots, 1)^{T}$ [21]. Therefore we have

$$
\dot{V} \leq \sum_{i=1}^{N} c_{i} \sum_{h \in \mathcal{N}_{i}} b_{i h}\left(v_{h 1}-v_{i 1}\right) \cdot v_{i 1}
$$

with equality if and only if we have heading consensus $\theta_{i j}=$ $\theta_{i k}$ for all agents within each group $i$. We now have

$$
\begin{aligned}
\dot{V} & \leq \sum_{i=1}^{N} c_{i} \sum_{h \in \mathcal{N}_{i}} b_{i h}\left(v_{h 1}-v_{i 1}\right) \cdot v_{i 1} \\
& =\sum_{i=1}^{N} c_{i} \sum_{h \in \mathcal{N}_{i}} b_{i h}\left(\dot{x}_{h 1} \dot{x}_{i 1}-\dot{x}_{i 1}^{2}+\dot{y}_{h 1} \dot{y}_{i 1}-\dot{y}_{i 1}^{2}\right) \\
& \leq \sum_{i=1}^{N} c_{i} \sum_{h \in \mathcal{N}_{i}} b_{i h}\left(\frac{\dot{x}_{h 1}^{2}+\dot{x}_{i 1}^{2}}{2}-\dot{x}_{i 1}^{2}+\frac{\dot{y}_{h 1}^{2}+\dot{y}_{i 1}^{2}}{2}-\dot{y}_{i 1}^{2}\right) \\
& =\sum_{i=1}^{N} c_{i} \sum_{h \in \mathcal{N}_{i}} b_{i h} \frac{1}{2}\left(\dot{x}_{h 1}^{2}-\dot{x}_{i 1}^{2}+\dot{y}_{h 1}^{2}-\dot{y}_{i 1}^{2}\right) .
\end{aligned}
$$

It is easy to verify that the set of edge functions $\left\{F_{i}^{h}=\right.$ $\left.\frac{1}{2}\left(\dot{x}_{h 1}^{2}-\dot{x}_{i 1}^{2}+\dot{y}_{h 1}^{2}-\dot{y}_{i 1}^{2}\right) \quad: \quad i, h \in V\left(\mathcal{G}_{B}\right)\right\}$ satisfies the cycle condition. Thus by Theorem 2 we have $\dot{V} \leq 0$. We also have $\dot{V}=0$ if and only if $\theta_{i j}=\theta_{i k}$ for all agents within each group $i$ and the velocities of the leaders of each group are identical $\left(v_{i 1}=v_{h 1}\right.$ for all group indices $i, h \in$ $\{1, \ldots, N\})$. Thus the system's configuration will converge to the largest invariant set $M$ contained in $\left\{(r, v): \theta_{11}=\right.$ $\cdots=\theta_{N n_{N}}$ and $\left.v_{11}=\cdots=v_{N 1}\right\}$. So we see that part (b) of Definition 2 is satisfied.

To see that part (a) is satisfied, consider the derivative of $P_{i j}$ along trajectories of the system (3). In $M$, we have

$$
\dot{P}_{i j}=\nabla_{r_{i j}} P_{i j} \cdot v_{i j}=\nabla_{r_{i j}} P_{i j} \cdot\left(-\nabla_{r_{i j}} P_{i j}\right) \leq 0 .
$$

Thus trajectories converge to a configuration corresponding to a local minimum of $P_{i j}$.

Compared with the complete network, the HPC scheme shows considerable savings in terms of the number of communication links, since the leader communication graph can be chosen arbitrarily, provided that it is strongly connected. This comes at the expense of the guarantee of collisionavoidance provided by the complete topology.

\section{Simulations}

To demonstrate our results numerically, we begin by randomly assigning initial positions and velocities to a set of 13 agents. Positions are assigned within a 10 by 10 area using a uniform distribution, and vertical and horizontal velocities are assigned uniformly in the range $[-1,1]$ (Figure 3a).

To group agents into clusters, we perform a $k$-means clustering [18] with the parameter $k$ set to 4 (Figure 3b). Each cluster is assigned a leader at random and any pair of distinct agents within a cluster is linked by the artificial potential function $P(x)=\frac{1}{x^{2}}+\log x^{2}$ that has a global minimum at $x=1$. The resulting formation is shown in Figure $3 \mathrm{~d}$. The distances between agents within each group converge to a value of approximately 1 which minimizes the potential functions, and the headings of all agents achieve a consensus. 


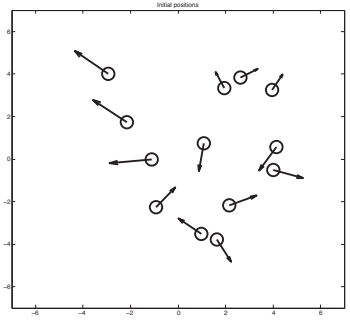

(a) Randomly generated positions

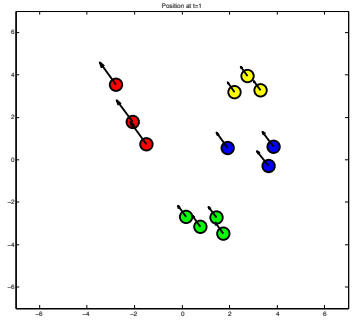

(c) Position at time $t=1$ of 13 agents at $t=0$

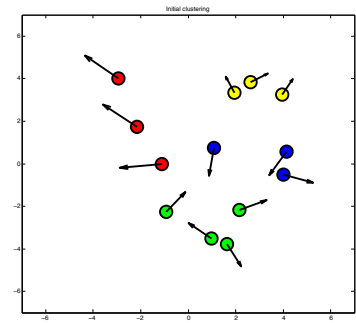

(b) Agents are grouped using a kmeans clustering

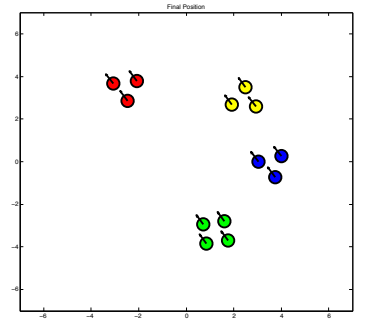

(d) Position at time $t=6$
Fig. 3

\section{ACKNOWLEDGMENTS}

This research is supported in part by grants from the Natural Sciences and Engineering Research Council of Canada (NSERC) and Canada Foundation for Innovation (CFI). MYL acknowledges the financial support from the NCE-MITACS. JM acknowledges the financial support of an NSERC USRA awarded at the University of Alberta.

\section{REFERENCES}

[1] T. Balch and R. C. Arkin, "Behavior-based formation control for multirobot teams," IEEE Transactions on Robotics and Automation, vol. 14, pp. 926-939, 1999.

[2] M. Egerstedt and X. Hu, "Formation constrained multi-agent control," IEEE Transactions on Robotics and Automation, vol. 17, no. 6, pp. $947-951,2001$

[3] J. Fax and R. Murray, "Information flow and cooperative control of vehicle formations," IEEE Transactions on Automatic Control, vol. 49, no. 9, pp. 1465 - 1476, 2004.

[4] L. Consolini, F. Morbidi, D. Prattichizzo, and M. Tosques, "Stabilization of a hierarchical formation of unicycle robots with velocity and curvature constraints," IEEE Transactions on Robotics, vol. 25, no. 5, pp. $1176-1184,2009$.

[5] R. Olfati-Saber and R. Murray, "Distributed structural stabilization and tracking for formations of dynamic multi-agents," in IEEE Conference on Decision and Control, 2002, pp. 209 - 215.

[6] H. Tanner, G. Pappas, and V. Kumar, "Leader-to-formation stability," IEEE Transactions on Robotics and Automation, vol. 20, no. 3, pp. 443 - 455, 2004.

[7] M. Arcak, "Passivity as a design tool for group coordination," IEEE Transactions on Automatic Control, vol. 52, pp. 1380 -1390, 2007.

[8] M. Y. Li and Z. Shuai, "Global-stability problem for coupled systems of differential equations on networks," Journal of Differential Equations, vol. 248, no. 1, pp. 1-20, 2010.

[9] J. Moon, Counting Labelled Trees, ser. Canadian Mathematical Monographs, vol. 1. Canadian Mathematical Congress, Montreal, 1970.

[10] D. E. Knuth, Fundamental Algorithms, ser. The Art of Computer Programming, vol, 1. Massachusetts: Addison-Wesley, 1997.

[11] A. Williams, S. Glavaski, and T. Samad, "Formations of formations: hierarchy and stability," in American Control Conference, 2004, pp. 2992-2997.
[12] D. Tsubakino and S. Hara, "Eigenvector-based intergroup connection of low rank for hierarchical multi-agent dynamical systems," Systems \& Control Letters, vol. 61, pp. $354-361,2012$.

[13] H. G. Tanner, A. Jabdabaie, and G. J. Pappas, "Stable flocking of mobile agents, Part I: Fixed topology," in IEEE Conference on Decision and Control, 2003, pp. 2010-2015.

[14] H. G. Tanner, A. Jadbabaie, and G. J. Pappas, "Stability of flocking motion," Technical Report, University of Pennsylvania, 2003.

[15] N. Leonard and E. Fiorelli, "Virtual leaders, artificial potentials and coordinated control of groups," in IEEE Conference on Decision and Control, 2001, pp. $2968-2973$.

[16] E. Rimon and D. Koditschek, "Exact robot navigation using artificial potential functions," IEEE Transactions on Robotics and Automation, vol. 8, pp. $501-518,1992$.

[17] W. A. Barbakh, Y. Wu, and C. Fyfe, "Review of clustering algorithms," in Non-Standard Parameter Adaptation for Exploratory Data Analysis. Springer, 2009, pp. 7-28.

[18] A. Jain, K. Murty, N. M, and P. J. Flynn, "Data clustering: a review," ACM Computing Surverys, vol. 31, pp. 264-323, 1999.

[19] B. J. Frey and D. Dueck, "Clustering by passing messages between data points," Science, vol. 315, pp. 972-976, 2007.

[20] R. A. Brualdi, Combinatorial Matrix Classes. Cambridge University Press, 2006.

[21] R. Olfati-Saber and R. M. Murray, "Consensus problems in networks of agents with switching topology and time-delays," IEEE Transactions on Automatic Control, vol. 49, pp. 1520-1533, 2004. 